\title{
RIBBON KNOT FAMILIES VIA STALLINGS' TWISTS
}

\author{
L. RICHARD HITT and DANIEL S. SILVER
}

(Received 18 May 1988; revised 29 November 1989)

Communicated by J. H. Rubinstein

\begin{abstract}
We invesiigate the effect on the Jones polynomial of a ribbon knot when two of its bands are twisted together. We use our results to prove that each of the three $S$-equivalence classes of genus 2 fibered doubly slice knots in $S^{3}$ can be represented by infinitely many distinct prime fibered doubly slice ribbon knots.
\end{abstract}

1980 Mathematics subject classification (Amer. Math. Soc.) (1985 Revision): 57 M 25.

Keywords and phrases: knot, Jones polynomial, fibered knot, doubly slice knot.

\section{Introduction}

In 1973 Burde and Neuwirth [18] conjectured that the number of fibered knots with a given Alexander polynomial is finite. Shortly afterward, Morton [16] and Stallings [20] independently proved that the conjecture is false. In fact, Stallings exhibited infinitely many distinct prime fibered ribbon knots $S_{2 n}, n \geq 0$, with the same Alexander polynomial as the square knot $3_{1} \#$ $\left(-3_{1}\right)$ (where $3_{1}$ denotes the right-hand trefoil) but with different Alexander modules.

The Jones polynomial is surprisingly twist-sensitive and can be used to distinguish Stallings' knots $S_{2 n}$ (see Section 3). Motivated by this consideration, we modify Stallings' construction to produce infinitely many distinct prime fibered doubly slice ribbon knots $K_{2 n}, n \in \mathbb{Z}$, that have the same Seifert

The second author was partially supported by a grant from the University of South Alabama Research and Public Service Council.

(C) 1991 Australian Mathematical Society 0263-6115/91 \$A2.00+0.00 
matrix (hence the same Alexander module) as the square knot and are also distinguished by their Jones polynomials. By combining our observations with the main result of [1] we obtain the following strengthened version of [3, Proposition 2]:

THEOREM 1. Each of the three $S$-equivalence classes of genus 2 fibered doubly slice knots can be represented by infinitely many distinct prime fibered doubly slice ribbon knots distinguished by their Jones polynomials.

The knots $K_{2 n}$ were originally proposed by Aitchison [1] who was able to distinguish an analogous collection, derived from $4_{1} \# 4_{1}$ (where $4_{1}$ denotes the figure eight knot) by examining stretching factors of pseudo-Anosov diffeomorphisms. Other examples of distinct fibered knots with the same Seifert matrix have been obtained by Morton [17] and Bonahon [5] while recent results of Long and Morton [15] provide the means to construct many more examples. In each case differentiation is achieved by considering the hyperbolic structure of the knots' exteriors.

In order to distinguish the knots $K_{2 n}$, we develop Kauffman's switching calculus for calculating his bracket invariant for double stranded links and doubles of knots. We then study the effect on the Jones polynomial of any ribbon knot when two of its bands are twisted together.

Much of this work was inspired by the results of Kanenobu [11], [12] . In [11] Kanenobu used the Jones polynomial and its 2-variable generalization [7] to complete a classification of fibered ribbon knots introduced in [10]. The knots, parameterized by integers $p$ and $q$, are fibered ribbon knots and are prime if $(p, q) \neq(0,0)$; their Alexander modules are determined by $p-q$.

A knot $K \subset S^{3}$ is doubly slice if $K=U \cap S^{3} \subset S^{4}$, where $U$ is an unknotted 2-sphere in $S^{4}$. The reader may wish to consult [19], [8], [21], [7] for definitions of other terms used and for background information about knots and the Jones polynomial.

Many results in this paper concerning knots can be stated more generally for links. For simplicity, we have chosen not to do this.

The authors are grateful to the referee for making several valuable suggestions, and to Professor Dale Rolfsen for suggesting the method of attack used in the proof of Corollary 3.2.

\section{Construction of $K_{n}$}

It is well-known that $K \#(-K)$ is a ribbon knot for any knot $K$. Also, if $K$ is fibered then so is $K \#(, K)$ (see [19] for example). Consider the fibered 


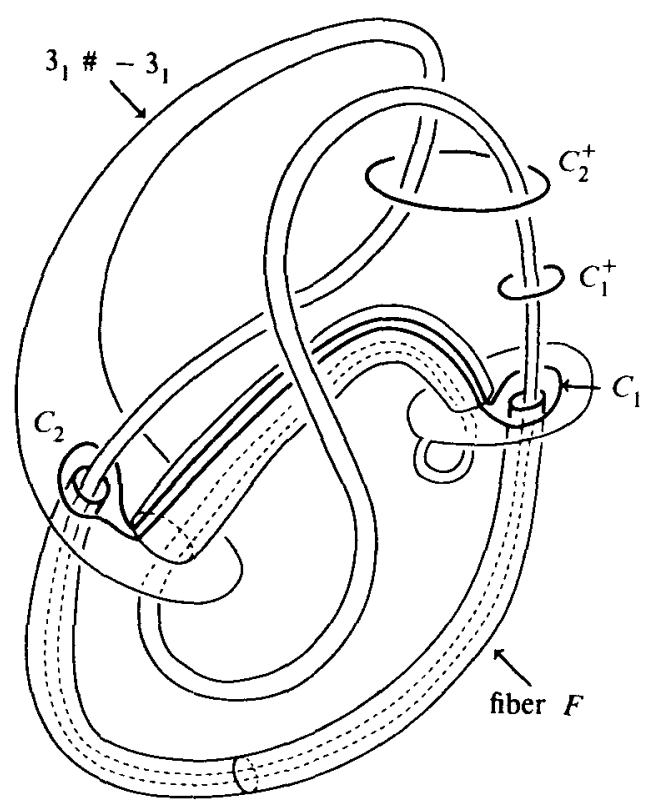

Figure 1

ribbon knot $3_{1} \#\left(-3_{1}\right)$ (the square knot) as it appears in Figure 1 together with a minimal-genus Seifert surface (hence a fiber) $F$. Each of the circles $C_{i}^{+}(i=1,2)$ is a normal push-off of a simple closed curve $C_{i}$ in $F$. Let $D_{i}$ denote the obvious disk with boundary $C_{i}$. Stallings' knots $S_{2 n}, n \geq 0$, can be obtained by giving $2 n$ right-hand half-twists to everything passing through $D_{1}$, as in Figure 2. We define $K_{n}, n \in \mathbb{Z}$, to be the knot obtained by giving $|n|$ half-twists to everything passing through $D_{2}$, as in Figure 2 .

By [20] the knots $K_{2 n}, n \in \mathbb{Z}$, are fibered since they are obtained from a fibered knot $3_{1} \#\left(-3_{1}\right)$ by Stallings' twists as described above. In fact, $K_{-1}$ is fibered: it can be obtained by performing Stallings' twists at two sites of the fibered knot $\left(-3_{1}\right) \# 4_{1}$ (see Figure 3). Since the knots $K_{2 n+1}, n \in \mathbb{Z}$, are obtained from $K_{-1}$ by Stallings' twists, they are also fibered. (The proof of Theorem 1 will contain another proof that the knots $K_{n}$ are fibered.)

The interest in our choice of curve $C_{2}$ in Figure 1 comes from the following observation in [1]. (See [17, pp. 214-215] also.)

Lemma 1. The knots $K_{2 n}, n \in \mathbb{Z}$, have the same Seifert matrix. Similarly for $K_{2 n+1}, n \in \mathbb{Z}$. 

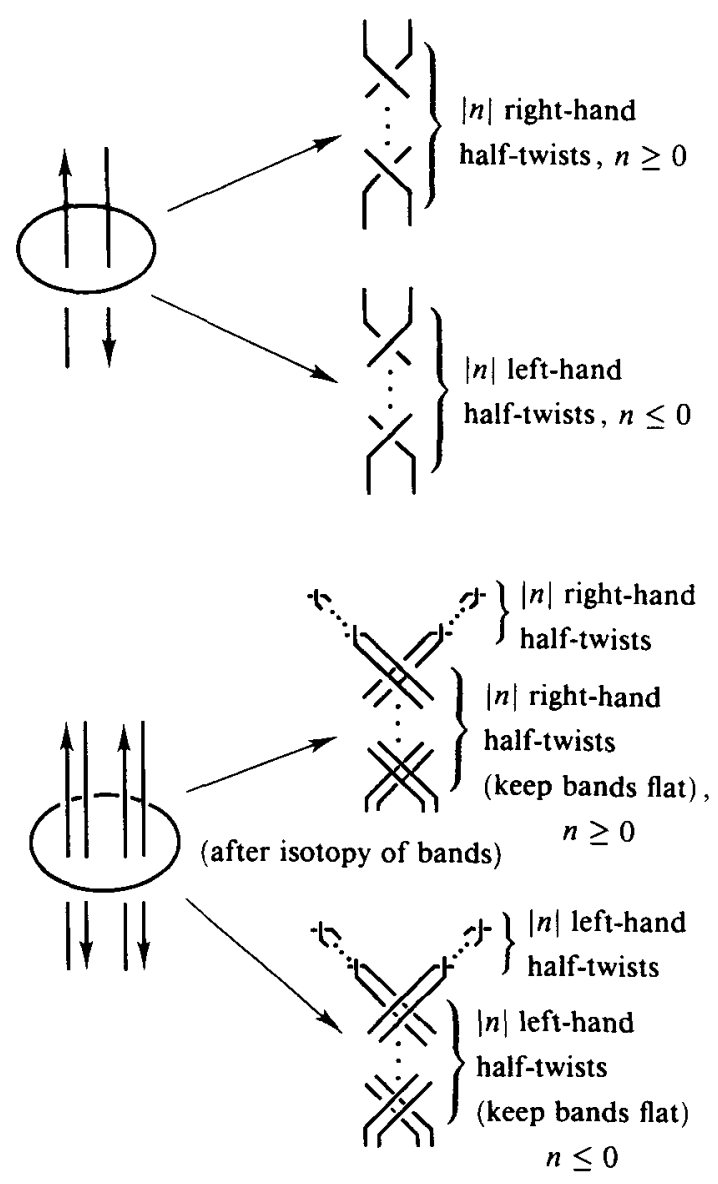

FIGURE 2

Proof (Aitchison). The circle $C_{2}^{+}$bounds a punctured torus disjoint from the fiber $F$. (This is easy to see in Figure 1 ; the torus is obtained by surgering $D_{2}$.) Choose a set of embedded circles $\left\{a_{i}\right\} \subset F$ representing a basis for $H_{1}(F ; \mathbb{Z})$, and push each circle off to obtain an embedded circle $a_{i}^{+}$in a neighborhood of $F$. The linking number of $a_{i}, a_{j}^{+}$is unchanged by twisting any even number of times everything that passes through $D$, since $a_{i}, a_{j}^{+}$ are both algebraically unlinked from $C_{2}$.

It follows that each of the knots $K_{2 n}, n \in \mathbb{Z}$, has the same Alexander polynomial as $K_{0}$, namely, $\left(t^{2}-t+1\right)\left(t^{-2}-t^{-1}+1\right)$. On the other hand, each of the knots $K_{2 n+1}, n \in \mathbb{Z}$, has Alexander polynomial $\left(t^{2}-t-1\right)\left(t^{-2}-\right.$ $\left.t^{-1}-1\right)$. The second assertion can be readily seen by computing the 1 relator presentation $\left(x, y: y=x y^{-2} x y^{2} x^{-1}\right)$ for $\pi_{1}\left(B^{4}-D\right)$, where $D$ is 


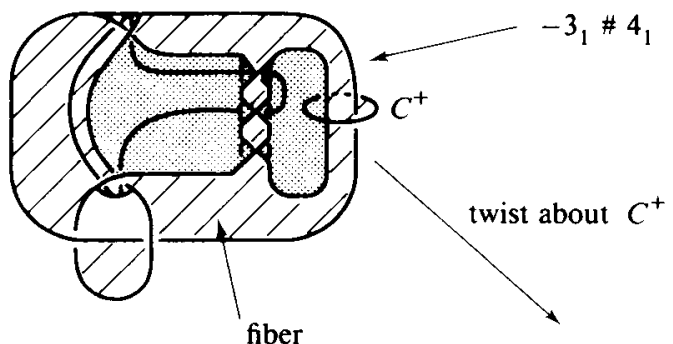

fiber

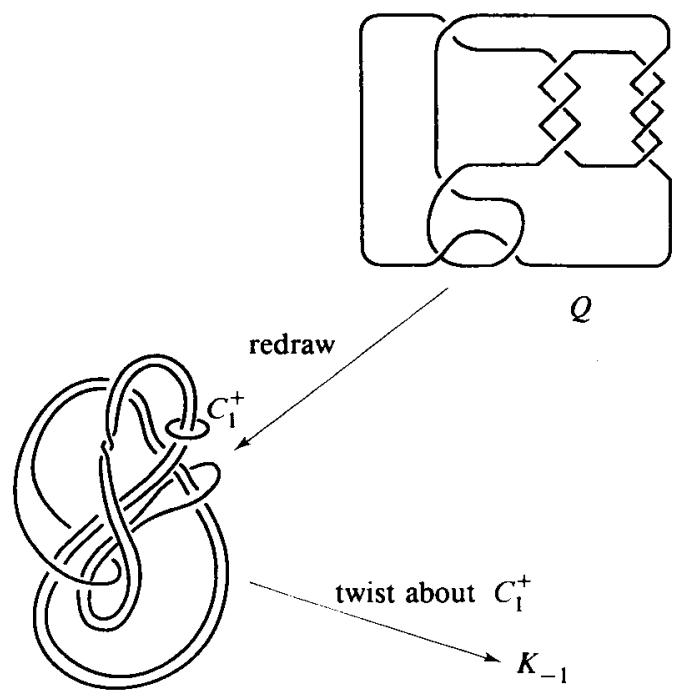

FIGURE 3

the obvious ribbon disk in $B^{4}$ with boundary $K_{-1}$, and noting that its Alexander polynomial is $t^{2}-t-1$. A standard duality argument involving order ideals (see [3, Proposition 1], for example) completes the argument.

\section{Jones polynomials of twisted ribbon knots}

Let the symbols [ $X],[X]$, and [ $\rangle$ ] represent three oriented links which are identical except near one point where they differ as depicted in the notation. Also let $V(L ; t)$ denote the Jones polynomial in the variable $t$ of a link $L$. Recall that the Jones polynomial satisfies the identity

$$
t^{-1} V([\times] ; t)-t V([X] ; t)+\left(t^{-1 / 2}-t^{1 / 2}\right) V([)(] ; t)=0
$$




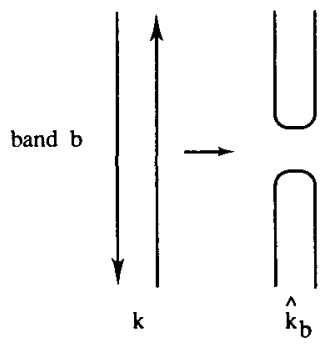

FIGURE 4

This equation, together with the condition that $V($ unknot; $t)=1$, uniquely defines the Jones polynomial of $L$. (See [9] for details.) In order to compute the Jones polynomials of the knots $K_{n}$ we need the following lemmas.

Let $k$ be any ribbon knot and consider a specific ribbon band presentation for $k$. Let $k(b, n)$ denote the new ribbon knot obtained by putting $|n|$ halftwists in a band $b$. (See Figure 2.) Let $\hat{k}_{b}$ denote the 2-component link that results when the band $b$ is broken, as in Figure 4 . If the knot $k$ has only one band, we will abbreviate $k(b, n)$ by $k(n)$. Notice that in this case, $\hat{k}_{b}$ is trivial. The following has been observed by Kanenobu [12].

LEMMA 2 (Ribbon band twisting). For any integer $n$,

(a) $V(k(b, n) ; t)=t^{n} V(k ; t)+\mu^{-1}\left(1+(-1)^{n-1} t^{n}\right) V\left(\hat{k}_{b} ; t\right)$, where $\mu^{-1}$ denotes the formal inverse of $\mu=-t^{-1 / 2}-t^{1 / 2}$;

(b) $V(k(b, 2 n) ; t)=t^{2 n} V(k ; t)+\mu^{-1}\left(1-t^{2 n}\right) V\left(\hat{k}_{b} ; t\right)$;

(c) $V(k(2 n) ; t)=t^{2 n} V(k ; t)-t^{2 n}+1$.

Let $\operatorname{Deg} V(k ; t)$ denote the largest exponent of $t$ in $V(k ; t)$ and let $\operatorname{deg} V(k ; t)$ denote the smallest exponent of $t$ in $V(k ; t)$. The following can be seen by straightforward calculation.

Corollary 2.1. If $V(k ; t) \neq \mu^{-1} V\left(\hat{k}_{b} ; t\right)$, then the knots $k(b, 2 n), n \in$ $\mathbb{Z}$, are distinct. Moreover, for $n>0$ and sufficiently large, $\operatorname{Deg} V(k(b, 2 n) ; t)$ is strictly increasing. Also, for $n>0$ and sufficiently large, $\operatorname{deg} V(k(b,-2 n) ; t)$ is strictly decreasing.

REMARKs. (1) The reader is cautioned that [12] employs an early definition of the Jones polynomial in which $t^{-1}$ replaces $t$ everywhere in (1).

(2) Stallings' knots $S_{2 n}$ have Jones polynomial

$$
V\left(S_{2 n} ; t\right)=t^{2 n}\left[\left(t+t^{3}-t^{4}\right)\left(t^{-1}+t^{-3}-t^{-4}\right)\right]-t^{2 n}+1 \text {. }
$$




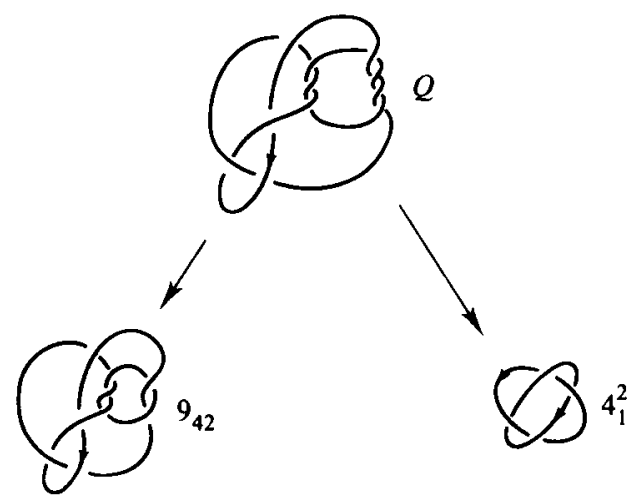

FIGURE 5

(3) For knots having a ribbon band presentation with only one band, the hypothesis $V(k ; t) \neq \mu^{-1} V\left(\hat{k}_{b} ; t\right)$ is equivalent to $V(k ; t) \neq 1$. In general, one may use Kauffman's bracket polynomial [13] to see that $V(k ; t) \neq$ $\mu^{-1} V\left(\hat{k}_{b} ; t\right)$ if and only if $V(k ; t) \neq V(k(b, 1) ; t)$.

(4) It can easily be seen that $K_{-1}=Q(-4)$, where $Q$ is depicted in Figure 3. By performing a single Conway move on $Q$ (Figure 5 ) we obtain the knot $9_{42}$ and one of the links $4_{1}^{2}$, the Jones polynomials of which can be easily computed or, with proper attention to orientation details, looked up in a table. From (1) it follows that $V(Q ; t)=t^{-5}-t^{-4}+t^{-3}-t^{-2}+1-t+t^{2}-t^{3}+t^{4}$ and using Lemma 2 we can immediately write down the Jones polynomial of $K_{-1}$ :

$$
V\left(K_{-1} ; t\right)=t^{-9}-t^{-8}+t^{-7}-t^{-6}-t^{-3}+t^{-2}-t^{-1}+2 .
$$

We will make use of this fact shortly.

If a ribbon knot is altered by switching a band crossing, we can find the Jones polynomial of the resulting (ribbon) knot, but we must work harder: Lemma 3 below is Kauffman's identity 3.2 [13] applied to the case of a ribbon band presentation of a knot at a site where one band crosses another.

LEMMA 3 (Ribbon band switching).

$$
\begin{aligned}
V([\mathrm{~N}] ; t)= & \left.V([\mathrm{~N}] ; t)+\left(t-t^{-1}\right)[V([\approx] ; t)-V([)] ; t)\right] \\
& +\left(t^{1 / 2}-t^{-1 / 2}\right)[V([\approx] ; t)+V([\approx] ; t) \\
& -V([) \div] ; t)-V([\gg][t)]
\end{aligned}
$$

ReMarks. (1) Kauffman's identity 3.2 [13] is stated in terms of his bracket puiynomial, which is a regular isotopy invariant. Following Kauffman, to 
obtain an isotopy invariant, we multiply the bracket polynomials by an appropriate factor (involving the writhe), and to obtain the Jones polynomial, Kauffman's variable is replaced by $t^{-1 / 4}$.

(2) Notice that twisting an even number of times and/or switching the bands of any ribbon knot produces a (ribbon) knot with the same Alexander polynomial as the original knot. (The original knot and the altered knot bound ribbon disks in $B^{4}$ with the same group.) Consequently, ribbon knots provide an inexhaustible supply of knots with the same Alexander polynomial but different Jones polynomials.

As an application of Lemma 3, consider any ribbon knot $k$ with a specific ribbon band presentation. Suppose that bands $b_{1}$ and $b_{2}$ (possibly the same) pass through a trivial circle $\Sigma$ disjoint from $k$, as in Figure 2 . Let $k$ denote the ribbon knot or link obtained by giving $|n|$ half-twists to everything passing through the obvious disk that $\Sigma$ bounds. (We continue to use the twist convention of Figure 2.) The following relation follows immediately from Lemma 3 and then Lemma 2:

$$
V\left(k_{n} ; t\right)=t^{-4} V\left(k_{n+2} ; t\right)+\left(t^{-3}-t^{-1}\right) V\left(k_{n+1} ; t\right)+C_{n},
$$

where

$$
\begin{aligned}
C_{n}= & \left(t-t^{-1}\right) V([\approx] ; t)+\left(t^{1 / 2}-t^{-1 / 2}\right)[V([\approx] ; t)+V([\gtrsim] ; t)] \\
& +(-t)^{n}\left(t^{-3 / 2}-t^{-1 / 2}\right)\left[V\left(\hat{k}_{b_{1}} ; t\right)+V\left(\hat{k}_{b_{2}} ; t\right)-V\left(\hat{k}_{b_{1}}^{\prime} ; t\right)-V\left(\hat{k}_{b_{2}}^{\prime} ; t\right)\right] \\
& +\mu^{-1}\left(t^{-7 / 2}-t^{-1 / 2}\right) V\left(\hat{k}_{b_{1} b_{2}} ; t\right) .
\end{aligned}
$$

Here $k^{\prime}$ denotes the ribbon knot or link obtained by replacing the twisting site $\uparrow \downarrow \uparrow$ in $k$ by . (Alternatively, $k^{\prime}$ is $k_{1}$ with one negative $\frac{1}{2}$-twist in each band $b_{1}, b_{2}$.) Also, $\hat{k}_{b_{1} b_{2}}$ denotes the link obtained from $k$ by breaking both bands $b_{1}, b_{2}$. In general, $C_{n}$ depends on $n$. However, when $b_{1}$ and $b_{2}$ are the same band $b$, then $C_{n}$ reduces to a constant:

$$
\begin{aligned}
C= & \left(t-t^{-1}\right) V([\approx] \approx t)+\left(t^{1 / 2}-t^{-1 / 2}\right)[V([\approx] ; t)+V([\approx] \\
& +\left(t-1+2 t^{-2}-t^{-3}-t^{-4}\right) \mu^{-1} V\left(\hat{k}_{b_{1}} ; t\right)+\left(2-2 t^{-2}\right) \mu^{-1} V\left(\hat{k}_{b_{2}} ; t\right) .
\end{aligned}
$$

The symbol $[\cdots]$ denotes the knot or link identical to $k$ except through $\Sigma$ where they differ as depicted. Notice that $C$ contains no fractional exponents since no other term in relation (2) does. Solving for $V\left(k_{n+2} ; t\right)$ we have

$$
V\left(k_{n+2} ; t\right)=t^{4} V\left(k_{n} ; t\right)+\left(t^{3}-t\right) V\left(k_{n+1} ; t\right)-t^{4} C \text {. }
$$

Using relations (2) and (3) we obtain the following result which is useful for distinguishing the knots $k_{n}$. 
Corollary 3.1. Let $k_{n}, n \in \mathbb{Z}$, be obtained by twisting two band segments of the same band $b$ of a ribbon knot $k$.

(1) If one of $1+\operatorname{Deg} V\left(k_{j} ; t\right), \operatorname{Deg} V\left(k_{j+1} ; t\right), 1+\operatorname{Deg} C$ is larger than the remaining two, then $\operatorname{Deg} V\left(k_{n} ; t\right)$ is strictly increasing for $n \geq j+1$.

(2) If one of $-4+\operatorname{deg} V\left(k_{-j+2} ; t\right),-3+\operatorname{deg} V\left(k_{-j+1} ; t\right), \operatorname{deg} C$ is less than the remaining two, then $\operatorname{deg} V\left(k_{-n} ; t\right)$ is strictly decreasing for $n \geq j-1$.

Corollary 3.2. Let $k_{n}, n \in \mathbb{Z}$, be obtained by twisting two band segments of the same band $b$ of a ribbon knot $k$. If $V(k ; t) \neq V\left(k_{1} ; t\right)$, then for sufficiently large $n, \operatorname{Deg} V\left(k_{n} ; t\right)$ is strictly increasing and $\operatorname{deg} V\left(k_{-n} ; t\right)$ is strictly decreasing.

Proof. For $n>0$, the polynomials $V_{n}=V\left(k_{n} ; t\right)$ can be found from the matrix equation

$$
\left(\begin{array}{c}
V_{n+1} \\
V_{n} \\
1
\end{array}\right)=A^{n}\left(\begin{array}{c}
V_{1} \\
V_{0} \\
1
\end{array}\right) \text {, where } A=\left(\begin{array}{ccc}
t^{3}-t & t^{4} & -t^{4} C \\
1 & 0 & 0 \\
0 & 0 & 1
\end{array}\right) \text {. }
$$

(Here we make use of relation (3).) Notice that $A$ has eigenvalues $\lambda_{1}=$ $t^{3}, \lambda_{2}=-t, \lambda_{3}=1$ with corresponding eigenvectors

$$
X_{1}=\left(\begin{array}{c}
t^{3} \\
1 \\
0
\end{array}\right), \quad X_{2}=\left(\begin{array}{c}
t \\
-1 \\
0
\end{array}\right), \quad X_{3}=\left(\begin{array}{c}
-t^{4} C \\
-t^{4} C \\
1+t-t^{3}-t^{4}
\end{array}\right) \text {. }
$$

Write

$$
\left(\begin{array}{c}
V_{1} \\
V_{0} \\
1
\end{array}\right)=f X_{1}+g X_{2}+h X_{3},
$$

for some $f, g, h$ in the field of fractions $\mathbb{Z}\left(t, t^{-1}\right)$ of $\mathbb{Z}\left[t, t^{-1}\right]$. Then

$$
\left(\begin{array}{c}
V_{n+1} \\
V_{n} \\
1
\end{array}\right)=t^{3 n} f X_{1}+(-t)^{n} g X_{2}+h X_{3} \text {. }
$$

Hence $V_{n}=t^{3 n} f+(-1)^{n+1} t^{n} g-h t^{4} C$. Clearly, if $f$ and $g$ are not both zero, then $\operatorname{Deg} V_{n}$ is strictly increasing for $n$ sufficiently large. But $f$ and $g$ are not both zero when $V_{0} \neq V_{1}$.

From relation (2), a similar argument applies to $\operatorname{deg} V\left(k_{-n} ; t\right)$ :

$$
\left(\begin{array}{c}
V_{-n} \\
V_{-n+1} \\
1
\end{array}\right)=B^{n}\left(\begin{array}{c}
V_{0} \\
V_{1} \\
1
\end{array}\right) \text {, where } B=\left(\begin{array}{ccc}
t^{-3}-t^{-1} & t^{-4} & C \\
1 & 0 & 0 \\
0 & 0 & 1
\end{array}\right) \text {. }
$$


The matrix $B$ has eigenvalues $\mu_{1}=t^{-3}, \mu_{2}=-t^{-1}, \mu_{3}=1$ with corresponding eigenvectors

$$
Y_{1}=\left(\begin{array}{c}
t^{-3} \\
1 \\
0
\end{array}\right), \quad Y_{2}=\left(\begin{array}{c}
t^{-1} \\
-1 \\
0
\end{array}\right), \quad Y_{3}=\left(\begin{array}{c}
C \\
C \\
1+t^{-1}-t^{-3}-t^{-4}
\end{array}\right)
$$

Write

$$
\left(\begin{array}{c}
V_{0} \\
V_{1} \\
1
\end{array}\right)=u Y_{1}+v Y_{2}+w Y_{3}
$$

for some $u, v, w \in \mathbb{Z}\left(t, t^{-1}\right)$. Then

$$
\left(\begin{array}{c}
V_{-n} \\
V_{-n+1} \\
1
\end{array}\right)=t^{-3 n} u Y_{1}+(-1)^{n} t^{-n} v Y_{2}+w Y_{3}
$$

Hence $V_{-n}=t^{-3(n+1)} u+(-1)^{n+1} t^{-n-1} v+w C$. Now $\operatorname{deg} V_{-n}$ is strictly decreasing for sufficiently large $n$, since $u$ and $v$ are not both zero when $V_{0} \neq V_{1}$.

REMARK. The condition in Corollary 3.2 that the two band segments are part of the same band can be eliminated. The same techniques, with a little more work, can show that the conclusion of the corollary holds for any two band segments.

We now return to the knots $K_{n}, n \in \mathbb{Z}$, constructed above. Calculation reveals that $C=t^{-6}-t^{-5}-t^{-4}-2 t^{-3}+2 t^{-1}+1+t-t^{2}$ (see Figure 6).
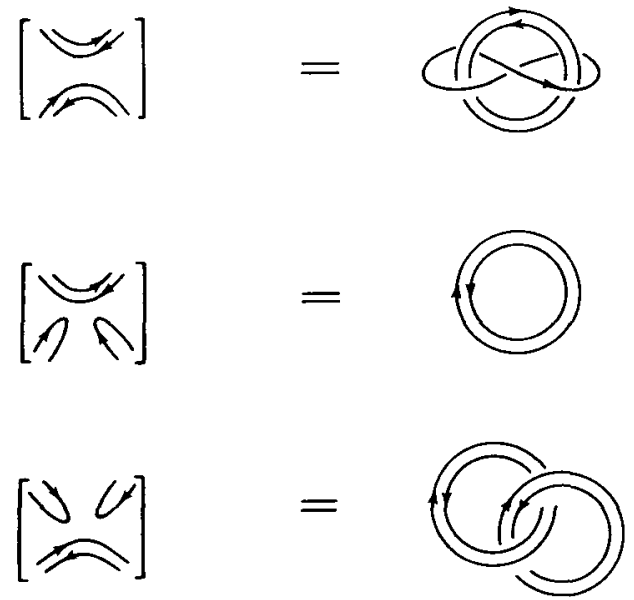

Figure 6 
Since $-3+\operatorname{deg} V\left(K_{-1} ; t\right)=-12$ is less than both $-4+\operatorname{deg} V\left(K_{0} ; t\right)=-7$ and $\operatorname{deg} C=-6$, it follows by Corollary 3.1 that $\operatorname{deg} V\left(K_{-n} ; t\right)$ is strictly decreasing for all $n \geq 1$. In fact, $\operatorname{deg} V\left(K_{-n} ; t\right)=-9-3(n-1)$ for $n \geq 1$.

The knots $K_{n}, n \geq 0$, can also be distinguished now if we use Corollary 3.1 , but extra care is needed since $1+\operatorname{Deg} V\left(K_{-1} ; t\right)=1, \operatorname{Deg} V\left(K_{0} ; t\right)=3$ and $1+\operatorname{Deg} C=3$. Using our calculation of $V\left(K_{-1} ; t\right)$ above together with relation (3) we find that

$$
V\left(K_{1} ; t\right)=t^{-5}-t^{-4}+t^{-3}-t^{-2}+1-t+t^{2}-t^{3}+t^{4}
$$

Applying (3) once more we obtain

$$
V\left(K_{2} ; t\right)=-t^{-4}+t^{-3}-t^{-2}+t^{-1}+2-t+2 t^{2}-3 t^{3}+2 t^{4}-2 t^{5}+t^{6} .
$$

Now since $\operatorname{Deg} V\left(K_{2} ; t\right)=6$ is larger than $1+\operatorname{Deg} V\left(K_{1} ; t\right)=5$ and $1+$ $\operatorname{Deg} C=3$ it follows by Corollary 3.1 that $\operatorname{Deg} V\left(K_{n} ; t\right)$ is strictly increasing for all $n \geq 0$. In fact, $\operatorname{Deg} V\left(K_{n} ; t\right)=6+3(n-2)$ for $n \geq 2$. In the Appendix we have included the Jones polynomial of $K_{n}$ for $|n| \leq 10$.

It now follows, of course, that all of the knots $K_{n}, n \in \mathbb{Z}$, are distinct. Furthermore, if $n \geq 1$ they are prime: if $K_{n}$ is composite, then it must be the connected sum of genus 1 fibered knots $3_{1},-3_{1}$, or $4_{1}$. An examination of Alexander polynomials shows that this is impossible. (See the second remark following Lemma 3.)

\section{Proof of Theorem 1}

Elementary matrix calculations as in [3], using results of [21], show that there are at most three S-equivalence classes of genus 2 fibered doubly slice knots in $S^{3}$. (See [3, Proposition 2].) Moreover, classes of such knots are distinguished by their Alexander polynomials: $\Delta_{1}(t)=\left(t^{2}-3 t+1\right)\left(t^{-2}-3 t^{-1}+\right.$ 1), $\Delta_{2}(t)=\left(t^{2}-t+1\right)\left(t^{-2}-t^{-1}+1\right), \Delta_{3}(t)=\left(t^{2}-t-1\right)\left(t^{-2}-t^{-1}-1\right)$. Aitchison [1] has produced infinitely many distinct knots as in the statement of Theorem 1 having Alexander polynomial $\Delta_{1}(t)$. Since $K_{2 n}$ and $K_{2 n+1}$ have Alexander polynomials $\Delta_{2}(t)$ and $\Delta_{3}(t)$, respectively, it suffices to show that these knots are doubly slice.

Casson and Gordon [6] have shown that the monodromy of any fibered ribbon knot extends over some handlebody. For the square knot more can be shown: Let $\Xi$ denote a genus 2 handlebody in $S^{3}$ obtained by thickening the fiber of a trefoil knot as in Figure 7. Let $D$ be a 2-disk and $w$ a nullhomologous curve on the boundary, as indicated. There exists an isotopy $h_{t}: S^{3} \rightarrow S^{3}, 0 \leq t \leq 1$, with the following properties.

(1) $h_{0}=$ identity. 

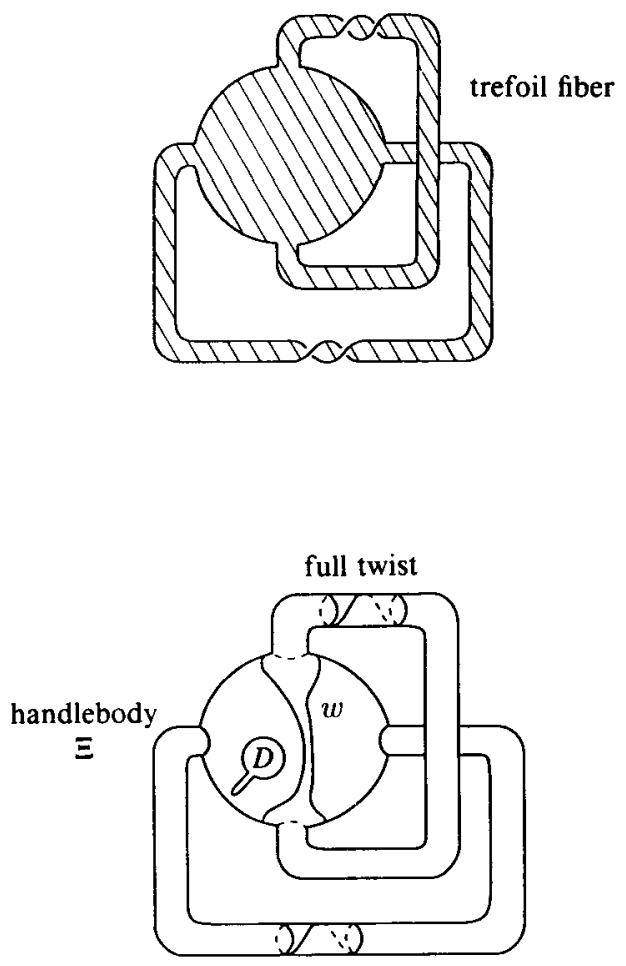

full twist

FIGURE 7

(2) $h_{1}$ restricted to $\Xi$ is a diffeomorphism $\phi: \Xi \rightarrow \Xi$.

(3) $h_{t}$ fixes a neighborhood in $\Xi$ of $D$ pointwise.

(4) The mapping torus $M(\phi)=\Xi \times_{\phi} S^{1}$ together with a 2-handle $\gamma$ attached along $D \times_{\phi} S^{1} \subset \partial M(\phi)$ is $B^{4}$.

(5) $\partial D \times\{0\} \subset \Xi \times\{0\}$ is the square knot.

(6) $w \times\{\varepsilon\}$ is isotopic to $C_{2}^{+}$.

In [1] Aitchison shows abstractly that an isotopy as above exists. In Figure 8 we give an explicit description of such an isotopy using handle slides. We keep track of framed representatives for a basis of $H_{1}(\Xi ; \mathbb{Z})$ for what follows.

Using techniques introduced by Akbulut and Kirby [4], Aitchison proves assertions (4)-(6) by constructing a handle decomposition for $M(\phi) \cup \gamma$ and simplifying. (See Figure 9.) Details of the construction can be found in [1]. (See also [2], [3].) If we now compose $\phi$ with $n$ ambient half-twists of $\Xi$ along $w$ before forming the mapping cylinder (Figure 10), the final result is $B^{4}$ with the knot $K_{n}$ on the boundary. 


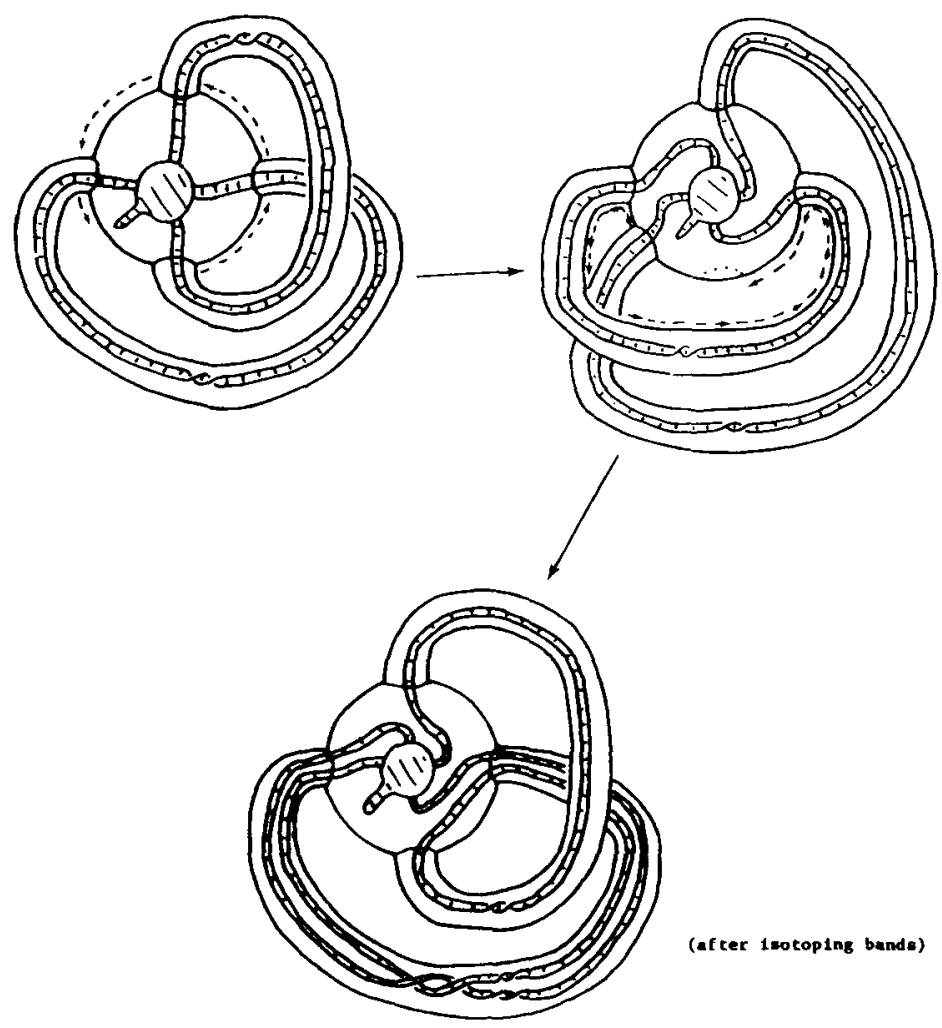

Figure 8

Clearly $D$ is a slice disk for $K_{n}$ in $B^{4}$. Also, since $\phi$ is the final map of an ambient isotopy, it's easy to see that $B^{4}-D \cong M(\phi)$ can embed in $S^{3} \times S^{1}$ inducing an isomorphism of integral first homology groups. By an observation of Levine [14, page 252] the disk knot $\left(B^{4}, D\right)$ is invertible. Consequently, $K_{n}$ is doubly slice.

Remarks. (1) Our differentiation of the knots $K_{n}, n \in \mathbb{Z}$, is much more effective than that obtainable by hyperbolic geometric techniques involving volume where one has to tolerate possible pairwise repetitions and conclusions for only sufficiently large $n$. (See [17, top of page 219].) However, the techniques of [5], used in [1], involve computing stretching factors from iterates of matrices, and when applicable are as effective as those considered here, since the possibility of repetition is excluded by monotonicity.

(2) In [1] Aitchison remarks that the boundary of $\Xi$ contains infinitely many null-homologous curves, other than $w$, along which we can twist $K_{0}$ 


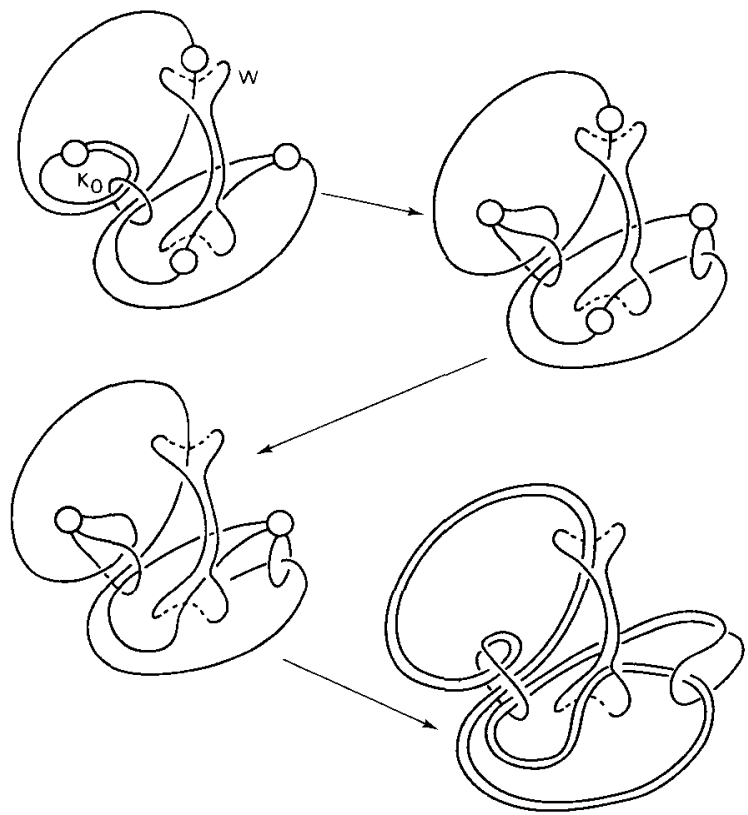

FIGURE 9

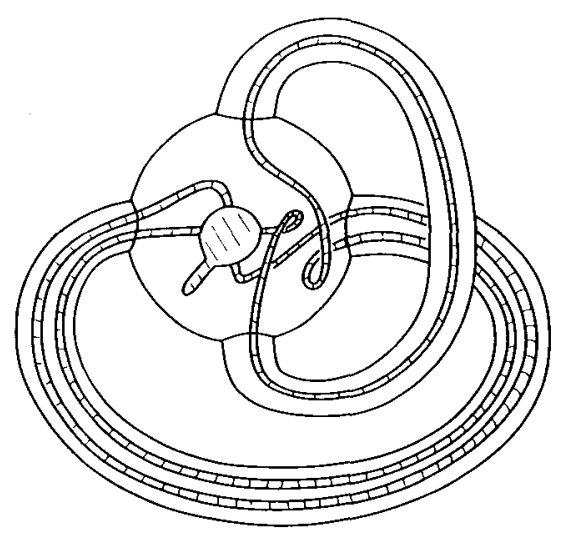

FIGURE 10

to construct potential families of knots as in Theorem 1. We expect that the members of many such families can be distinguished using the techniques above. 


\section{Questions}

(1) Let $k \subset S^{3}$ be any fibered knot with fiber $F$ and let $c$ be any essential simple closed curve in $F$ such that $c$ is unknotted in $S^{3}$ with self-linking number zero. Let $k_{n}$ denote the fibered knot obtained from $k$ by Stallings' twists (n times) along $c$, twisting always in the same direction. Is either the maximum deg ree or the minimum deg ree of $V\left(k_{n} ; t\right)$ a strictly monotone function for sufficiently large $n$ ?

Long and Morton [15] have shown that if $k$ is a hyperbolic knot, then for all sufficiently large $n$ the 3-manifolds obtained by $n$-framed surgery on $k$ are hyperbolic with strictly increasing (bounded) hyperbolic volumes.

(2) Does the Jones polynomial have special geometric significance for fibered hyperbolic knots?

(3) In [3] it was proved that if $f(t)=a_{d} t^{d}+\cdots+a_{1} t+a_{0}$ is any integral polynomial such that $a_{0} a_{d}=1$ and $f(1)= \pm 1$ then there exists a fibered (ribbon) doubly slice knot with Alexander polynomial $f(t) f\left(t^{-1}\right)$. Can twisting produce infinitely many such knots with identical Seifert matrices and distinct Jones polynomials?

\section{Appendix}

Using only the defining relation (1) of the Jones polynomial, we calculated the polynomials for the knots $K_{n}$ for $|n| \leq 10$. We wish to thank Peter Pacheco for providing a computer program which was of assistance, and to thank the Alabama Supercomputer Center for providing the necessary time on the Alabama Supercomputer Network Cray X-MP/24 for the calculations.

$$
\begin{aligned}
V\left(K_{10} ; t\right)= & t^{30}-t^{29}-t^{25}+t^{24}-t^{13}+2 t^{12}-3 t^{11}+3 t^{10}-2 t^{9}+2 t^{8}-t^{7}+t^{5}-t^{4}-t^{2} \\
& +2 t-1+2 t^{-1}-t^{-2} \\
V\left(K_{9} ; t\right)= & t^{27}-t^{26}-t^{22}+t^{21}+t^{12}-2 t^{11}+3 t^{10}-3 t^{9}+2 t^{8}-2 t^{7}+t^{6}-t^{4}+t^{3}-t^{2} \\
& +2 t-1+2 t^{-1}-t^{-2} \\
V\left(K_{8} ; t\right)= & t^{24}-t^{23}-t^{19}+t^{18}-t^{11}+2 t^{10}-3 t^{9}+3 t^{8}-2 t^{7}+2 t^{6}-t^{5}+t^{3}-2 t^{2} \\
& +2 t-1+2 t^{-1}-t^{-2} \\
V\left(K_{7} ; t\right)= & t^{21}-t^{20}-t^{16}+t^{15}+t^{10}-2 t^{9}+3 t^{8}-3 t^{7}+2 t^{6}-2 t^{5}+t^{4}-2 t^{2}+3 t-1 \\
& +2 t^{-1}-t^{-2} \\
V\left(K_{6} ; t\right)= & t^{18}-t^{17}-t^{13}+t^{12}-t^{9}+2 t^{8}-3 t^{7}+3 t^{6}-2 t^{5}+2 t^{4}-t^{3}-t^{2}+3 t-2 \\
& +2 t^{-1}-t^{-2}
\end{aligned}
$$




$$
\begin{aligned}
& V\left(K_{5} ; t\right)=t^{15}-t^{14}-t^{10}+t^{9}+t^{8}-2 t^{7}+3 t^{6}-3 t^{5}+2 t^{4}-2 t^{3}+2 t-2+3 t^{-1}-t^{-2} \\
& V\left(K_{4} ; t\right)=t^{12}-t^{11}-2 t^{7}+3 t^{6}-3 t^{5}+3 t^{4}-2 t^{3}+t^{2}+t-1+3 t^{-1}-2 t^{-2} \\
& V\left(K_{3} ; t\right)=t^{9}-t^{8}+t^{6}-2 t^{5}+2 t^{4}-2 t^{3}+t^{2}+2 t^{-1}-2 t^{-2}+t^{-3} \\
& V\left(K_{2} ; t\right)=t^{6}-2 t^{5}+2 t^{4}-3 t^{3}+2 t^{2}-t+2+t^{-1}-t^{-2}+t^{-3}-t^{-4} \\
& V\left(K_{1} ; t\right)=t^{4}-t^{3}+t^{2}-t+1-t^{-2}+t^{-3}-t^{-4}+t^{-5} \\
& V\left(K_{0} ; t\right)=-t^{3}+t^{2}-t+3-t^{-1}+t^{-2}-t^{-3} \\
& V\left(K_{-1} ; t\right)=2-t^{-1}+t^{-2}-t^{-3}-t^{-6}+t^{-7}-t^{-8}+t^{-9} \\
& V\left(K_{-2} ; t\right)=-t^{2}+t+1-t^{-1}+2 t^{-2}-2 t^{-3}+2 t^{-4}-t^{-5}+t^{-6}-t^{-8}-t^{-11}+t^{-12} \\
& V\left(K_{-3} ; t\right)=-t^{2}+2 t+2 t^{-2}-3 t^{-3}+2 t^{-4}-2 t^{-5}+t^{-6}-t^{-8}+2 t^{-9}-t^{-10}-t^{-14} \\
& +t^{-15} \\
& V\left(K_{-4} ; t\right)=-t^{2}+2 t-1+t^{-1}+t^{-2}-3 t^{-3}+3 t^{-4}-2 t^{-5}+2 t^{-6}-t^{-7}+t^{-9}-t^{-10} \\
& +t^{-12}-t^{-13}-t^{-17}+t^{-18} \\
& V\left(K_{-5} ; t\right)=-t^{2}+2 t-1+2 t^{-1}-2 t^{-3}+3 t^{-4}-3 t^{-5}+2 t^{-6}-2 t^{-7}+t^{-8}-t^{-10}+t^{-11} \\
& +t^{-15}-t^{-16}-t^{-20}+t^{-21} \\
& V\left(K_{-6} ; t\right)=-t^{2}+2 t-1+2 t^{-1}-t^{-2}-t^{-3}+2 t^{-4}-3 t^{-5}+3 t^{-6}-2 t^{-7}+2 t^{-8}-t^{-9} \\
& +t^{-11}-t^{-12}+t^{-18}-t^{-19}-t^{-23}+t^{-24} \\
& V\left(K_{-7} ; t\right)=-t^{2}+2 t-1+2 t^{-1}-t^{-2}+t^{-4}-2 t^{-5}+3 t^{-6}-3 t^{-7}+2 t^{-8}-2 t^{-9} \\
& +t^{-10}-t^{-12}+t^{-13}+t^{-21}-t^{-22}-t^{-26}+t^{-27} \\
& V\left(K_{-8} ; t\right)=-t^{2}+2 t-1+2 t^{-1}-t^{-2}-t^{-5}+2 t^{-6}-3 t^{-7}+3 t^{-8}-2 t^{-9}+2 t^{-10} \\
& -t^{-11}+t^{-13}-t^{-14}+t^{-24}-t^{-25}-t^{-29}+t^{-30} \\
& V\left(K_{-9} ; t\right)=-t^{2}+2 t-1+2 t^{-1}-t^{-2}+t^{-6}-2 t^{-7}+3 t^{-8}-3 t^{-9}+2 t^{-10}-2 t^{-11} \\
& +t^{-12}-t^{-14}+t^{-15}+t^{-27}-t^{-28}-t^{-32}+t^{-33} \\
& V\left(K_{-10} ; t\right)=-t^{2}+2 t-1+2 t^{-1}-t^{-2}-t^{-7}+2 t^{-8}-3 t^{-9}+3 t^{-10}-2 t^{-11}+2 t^{-12} \\
& -t^{-13}+t^{-15}-t^{-16}+t^{-30}-t^{-31}-t^{-35}+t^{-36}
\end{aligned}
$$

\section{References}

[1] I. R. Aitchison, 'Isotoping and twisting knots and ribbons,' (Ph.D. Dissertation, Univ. of California, Berkeley, 1984).

[2] I. R. Aitchison and J. H. Rubinstein, 'Fibered knots and involutions on homotopy spheres,' Four-Manifold Theory, pp. 1-74, (Amer. Math. Soc., Providence, R.I., 1985). 
[3] I.R. Aitchison and D.S. Silver, 'On fibering certain ribbon disk pairs,' Trans. Amer. Math. Soc. 306 (1988), 529-551.

[4] S. Akbulut and R. Kirby, 'An involution of $S^{4}$;'Topology 18 (1970), 75-81.

[5] F. Bonahon, 'Ribbon fibred knots, cobordism of surface diffeomorphisms, and pseudoAnosov diffeomorphisms,' Math. Proc. Cambridge Phil. Soc. 94 (1983), 235-251.

[6] A. J. Casson and C. M. Gordon, 'A loop theorem for duality spaces and fibered ribbon knots,' Invent. Math. 74 (1983), 119-137.

[7] P. Freyd, D. Yetter, J. Hoste, W. B. R. Lickorish, K. Millett, and A. Ocneanu, 'A new polynomial invariant of knots and links,' Bull. Amer. Math. Soc. 12 (1985), 239-246.

[8] C. M. Gordon, 'Some aspects of classical knot theory,' Knot Theory, (Lecture Notes in Math. 685, Springer-Verlag, 1978).

[9] V. F.R. Jones, 'A new knot polynomial and von Neumann algebras,' Notices Amer. Math. Soc. 33 (1986), 219-225.

[10] T. Kanenobu, 'Module d'Alexander des noeuds fibrés et polynôme de Hosokawa des lacements fibrés,' Math. Sem. Notes Kobe Univ. 9 (1981), 75-84.

[11] , 'Infinitely many knots with the same polynomial invariant,' Proc. Amer. Math. Soc. 97 (1986), 158-162.

[12] , 'Examples on polynomial invariants of knots and links,' Math. Ann. 275 (1986), 555-572.

[13] L. H. Kauffman, 'State models for knot polynomials,' preprint.

[14] J. Levine, 'Doubly slice knots and doubled disk knots,' Michigan Math. J. 30 (1983), 249-256.

[15] D. D. Long and H. R. Morton, 'Hyperbolic 3-manifolds and surface automorphisms,' Topology 25 (1986), 575-583.

[16] H. R. Morton, 'Infinitely many fibered knots having the same Alexander polynomial,' Topology 17 (1978), 101-104.

[17] , 'Fibered knots with a given Alexander polynomial,' L'Enseignment Math. 31 (1983), 205- 222.

[18] L. P. Neuwirth, 'The status of some problems related to knot groups,' Topology Conference VPISU, (Lecture Notes Math 375, pp. 209-230, Springer-Verlag, 1974).

[19] D. Rolfsen, Knots and Links, (Math. Lecture Ser. 7, Publish or Perish, Berkeley, 1976).

[20] J. Stallings, 'Constructions of fibered knots and links,' Proc. Sympos. Pure Math., vol. 32, pp. 55-60, (Amer. Math. Soc., Providence, R.I., 1978).

[21] D. W. Sumners, 'Invertible knot cobordisms,' Comment. Math. Helv. 46 (1971), 240256.

University of South Alabama

Mobile, Alabama 36688

U.S.A. 